\title{
Localization of Pelvic Anatomical Coordinate System Using US/Atlas Registration for Total Hip Replacement
}

\author{
Pezhman Foroughi $^{1}$, Danny Song ${ }^{1}$, Gouthami Chintalapani ${ }^{1}$, \\ Russell H. Taylor ${ }^{1}$, and Gabor Fichtinger ${ }^{1,2}$ \\ 1 Johns Hopkins University, Baltimore, MD, USA \\ ${ }^{2}$ Queen's University, Kingston, Canada \\ \{pezhman, greddy, rht\}@cs.jhu.edu, dsong2@jhmi.edu, gabor@cs.queensu.ca
}

\begin{abstract}
In Total Hip Replacement (THR) procedures, misalignment of the acetabular component can lead to dislocation and impingement. For the successful alignment of acetabular component, precise estimation of pelvic anatomical coordinate system is necessary. Conventional navigation systems use CT scan or fluoroscopy, or involve implanted bone fiducials or invasive probing of bony landmarks to locate the anatomical coordinate. In this paper, an ultrasound-based approach is proposed that exploits prior knowledge about the anatomy of the pelvis in the form of a $3 \mathrm{D}$ surface atlas. Tracked ultrasound images are utilized to extract sample points from the surface of the pelvis. A generic coordinate system in the specific patient is localized by registering these points to a statistical atlas of the pelvis in which a canonical anatomical coordinate system had been defined. This technique has been evaluated using simulation, dry bone, and cadaver experiments and was able to localize the anatomical coordinate system with the accuracy of about 1 degree.
\end{abstract}

Keywords: Orthopedic surgery, Statistical atlas, Ultrasound, Registration, Navigation, Total hip replacement.

\section{Introduction}

Accurate alignment of the acetabular component is a critical step in THR procedures. Misalignment of the acetabular component can lead to dislocation and impingement increasing the chance of the need for a revision surgery [12]. Navigation systems are proved to be successful in aligning the acetabular component [1. For placement of acetabular component, conventional navigation systems require intra-operative fluoroscopy or pre-operative $\mathrm{CT}$ of the bone or might involve invasive probing of bony landmarks with a tracked pointer.

In contrast, we propose an ultrasound-based approach which does not require fluoroscopy or a CT scan of the patient. Ultrasound is a safe, effective, and affordable intra-operative imaging tool in abdominal surgery, and it has begun to find its way to orthopaedic surgery as well. By obviating the need for preoperative $\mathrm{CT}$ and intra-operative fluoroscopy, ultrasound fits well with recent

D. Metaxas et al. (Eds.): MICCAI 2008, Part II, LNCS 5242, pp. 871-879, 2008.

(C) Springer-Verlag Berlin Heidelberg 2008 
trends in joint arthroplasty. Ultrasound adds little time to a clinical procedure, as tens of images can be acquired in a matter of seconds. Recently available portable ultrasound units provide good image quality, with little intrusion to the operating theater. (To specifically stress on this point, in this work we used a low-cost laptop ultrasound system.)

Recently, ultrasound was successfully coupled with OrthoPilot navigation system (B. Braun Aesculap, Tuttlingen, Germany) to measure length, axes, and torsion of a plastic leg model [3]. In 4], ultrasound based localization of bony landmarks was compared to pointer palpation based technique. Similar results of cup inclination values were reported for both methods. Interpretation and localization of bony structures in ultrasound is subjective, time consuming, and prone to error. These issues demand quantitative computational approaches that promise to transform ultrasound into a generic navigation tool in orthopedic surgical guidance. To this end, our methodology exploits prior knowledge about the anatomy of the pelvis in the form of a three-dimensional surface atlas. We collect tracked ultrasound images from the pelvis and extract sample points. We register these points to a statistical atlas of the pelvis in which a canonical anatomical coordinate system had been defined by the surgeon, and thus localize this generic coordinate system in the specific patient.

There is a large body of scholarly work dedicated to statistical shape models [5]. The feasibility of ultrasound-based reconstruction of the surface model of proximal femur using a dense point distribution model was evaluated in [6] Although the reported results are less accurate than those obtained by pointerbased approach, the high potential of ultrasound-based techniques is well recognized. It is assumed that after the manual initialization and affine registration of the points to the mean shape, the final pose is good enough for final mode recovery. With this assumption, in [7], the mode weights are directly calculated by differentiating the cost function.

In a similar work, a statistical shape model of femur was instantiated by registering the surface points manually segmented from ultrasound images to the statistical model 8. For each iteration of the registration, the weights for five modes of variation were determined after Iterative Closest Point (ICP) optimization. Furthermore, center of rotation of the femoral head was incorporated in the process as an additional constraint. Cadaver validation of this work was presented in 9, where two pelvises and three femurs of cadavers were reconstructed. The results show global registration accuracy of about $3 \mathrm{~mm}$ for femurs and $5 \mathrm{~mm}$ for pelvises. The cadavers are moved during image acquisition in order to collect images from all parts of bony anatomy accessible by ultrasound. The most important distinction between this work and our approach is that we collect the ultrasound images only from specific regions of bony anatomy with the purpose of finding the anatomical coordinate system. Moreover, we have adapted a faster optimization approach with a richer statistical atlas that has larger number of modes of variation to achieve better accuracy. Finally, we employ the outcomes of our previous work for an automatic initialization and ultrasound segmentation. A detailed description of our technique is presented in the following section. 


\section{Methodology}

Our ultimate goal is to find the anatomical coordinate system of the patient's pelvis. We have adapted the definition of pelvic coordinate system which is described in [10] and is successfully used in a hip navigation system, "HipNav" (CASurgica, Pittsburgh, PA). Nikou et. al [10] also illustrated the rationales for their choices of coordinate systems for pelvis, femur, and prosthetic components as well as the relations among these coordinate systems.

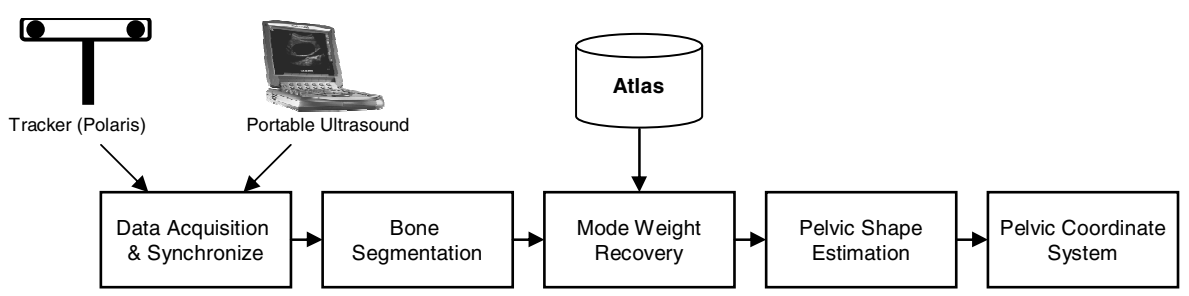

Fig. 1. The block diagram of the proposed system

The first step is to acquire tracked ultrasound images of pelvis. Four landmarks are used to define the coordinate system namely "the maximally anterior left and right iliac spine points, and the maximally anterior left and right pubis symphysis points [10]". Accordingly, the images are collected from the left and right iliac crest containing the anterior superior iliac spines, and the body of the pubis containing pubic tubercles. The surface of the bone is segmented out from ultrasound images using our bone segmentation method introduced in [1]. The segmentation is based on the appearance of bone surface in ultrasound images and dynamic programming optimization. The user can discard the images in which the bone surface cannot be reliably detected. Applying the tracking information to the segmentation output gives us a number of contours in $3 \mathrm{D}$ space as visualized in Figure 2(a).

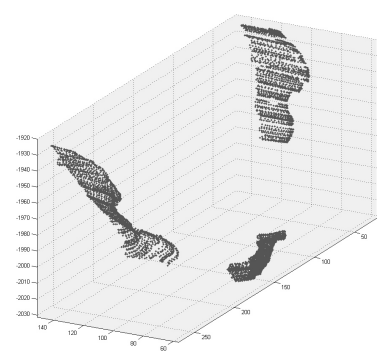

(a) Segmentation outcome

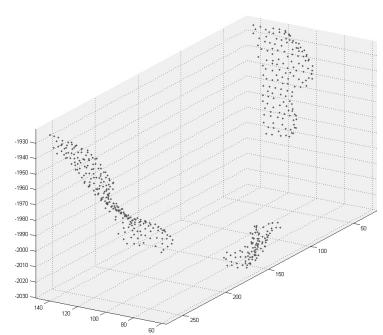

(b) Random sampling

Fig. 2. Even distribution of the sample points is achieved by randomly sampling the results of segmentation of ultrasound images 
The ultrasound images are collected while the transducer is being swept over the region of interest. Due to nonuniform motion in freehand data collection, several images could be collected from almost the same area while gaps are left behind in other parts as visible in Figure 2(a). In order to achieve evenly distributed points, the contours are randomly sampled (see Figure 2(b)]. The samples are not allowed to be closer than $3 \mathrm{~mm}$ to each other in order to decrease the amount of redundant information and speed up the program without loosing accuracy. Random sampling also removes the possible bias towards a region where data is densely collected.

The collected sample points are used to instantiate the surface atlas of pelvis. The atlas of pelvis is a statistical shape model constructed from CT scans of healthy patients and contains a mean shape of pelvis and several variational modes. Detailed information about construction of this atlas can be found in [12. When the weights for variational modes are recovered, the shape of the pelvis can simply be retrieved as follows:

$$
m^{*} \approx m_{0}+\sum_{i=1}^{n} \lambda_{i} m_{i}
$$

where $m^{*}$ represents the exact real location of vertices of the model of pelvis, $m_{0}$ and $m_{i}$ symbolize the mean shape and $i$ th mode of variation respectively, and $\lambda_{i}$ represents the weight for the $i$ th mode. The number of modes, $n$, is set to 15 since that is shown to be enough to accurately reconstruct the pelvis [12. The first step to recover the modes weights is to rigidly register the extracted sample points from ultrasound to the mean shape of the atlas. Initially, the points are transformed to a close proximity of the corresponding projected points on the mean shape based on our previous work published in [13. This work includes a full study of sensitivity to initialization error. After the initialization step, standard ICP is employed to obtain the final registration. The mode weights are calculated using a mode matching scheme similar to that of [14. At iteration $t+1$, the current instance of the atlas, $m^{t}$, is generated having the current mode weights, $\lambda_{i}^{t}$, using Equation (1). The current position of sample points $d^{t}$ is calculated as $d^{t}=F^{t} \bullet d^{0}$, where $F^{t}$ and $d^{0}$ represent the current registration transformation and the initial position of sample points respectively. The closest points on the surface of current instance to $d^{t}$ is computed and converted to the barycentric coordinates of the corresponding triangles on the mesh model. Using the barycentric area coefficients, the mean shape for the closest points, $q_{0}^{t}$ and the modes of variation for the closest points, $q_{i}^{t}$ are computed.

With the assumption of small change in rotation and translation, the updated registration transformation, $F^{t+1}$, can be estimated as follows:

$$
F^{t+1}=\left[\begin{array}{cc}
\left(I+s k\left(\alpha^{t+1}\right)\right) & \epsilon^{t+1} \\
0 & 1
\end{array}\right] \bullet F^{t},
$$

where $\alpha^{t+1}$ and $\epsilon^{t+1}$ are a small rotation vector and a small translation vector respectively. $I+s k\left(\alpha^{t+1}\right)$ estimates a small rotation, where $s k()$ is the $3 \times 3$ 
skew-symmetric matrix. With this linearization, $\lambda_{i}^{t+1}$ is determined from the following system of equations:

$$
s k\left(\alpha^{t+1}\right) d^{t}+\epsilon^{t+1}-\sum_{i=1}^{n} \lambda_{i}^{t+1} q_{i}^{t}=q_{0}^{t}-d^{t} .
$$

This linear system is easily solved for $\alpha^{t+1}, \epsilon^{t+1}$, and $\lambda_{i}^{t+1}$ in a least squares sense using QR decomposition. This formulation increases the convergence rate as the optimization of the mode weights and the transformation parameters are combined in one step. The stopping criterion is the combination of a minimal change in the optimization variables or reaching a maximum number of iterations. We employ two mechanisms that suppress the effect of outliers: first, the large number of acquired images followed by random sampling lowers the percentage of outliers; second, in the iteration of the algorithm, the points with high deviation are excluded.

In order to recover the pelvic coordinate frame, the resulting instance of the atlas is transformed back into the ultrasound coordinate system. A general pelvic coordinate system is defined by selecting the four landmarks described earlier on the "mean shape". Since the meshes of the mean shape and any instance of the atlas correspond to each other, the four landmarks are automatically transfered to the estimated shape. The coordinate system is then computed from these landmarks as illustrated in [10].

\section{$3 \quad$ Experiments and Results}

The method was initially evaluated using simulation experiments. The two advantages of simulation studies are the availability of a solid ground truth and easily controllable model and noise parameters. For simulation, an instance of the atlas was created, and the surface of the model was sampled at the areas of interest. A randomly generated uniform noise of maximum $2 \mathrm{~mm}$ was then added to the three coordinates of each sample. Afterwards, a small random transformation was applied to the points with maximum rotation parameters of 5 degrees and maximum translation parameters of $5 \mathrm{~mm}$. Our method was then used to reconstruct the pelvis and find the pelvic coordinate system. This experiment was repeated 100 times, yielding sub-mm and sub-degree accuracies reported in Table 1

Table 1. Estimate errors of the measured anatomical coordinate system (For the simulation experiment, the average error is reported)

\begin{tabular}{|c|c|c|c|c|c|c|}
\hline \multirow{2}{*}{ Experiment } & \multicolumn{5}{|c|}{ Error of anatomical coordinate system localization } \\
\cline { 2 - 7 } & $\alpha$ (degrees) & $\beta$ (degrees) & $\gamma$ (degrees) & $x(\mathrm{~mm})$ & $y(\mathrm{~mm})$ & $z(\mathrm{~mm})$ \\
\hline \hline Simulation & 0.4 & 0.1 & 0.4 & 0.1 & 0.4 & 0.5 \\
\hline Dry Bone & 0.8 & 0.8 & 0.7 & 1.9 & 1.8 & 0.3 \\
\hline Cadaver one & 0.7 & 0.7 & 1.0 & 0.9 & 2.0 & 2.3 \\
\hline Cadaver two & 1.3 & 1.4 & 0.4 & 1.3 & 0.4 & 3.3 \\
\hline
\end{tabular}


A dry bone experiment and two cadaver experiments were conducted in order to evaluate the accuracy of proposed technique in a realistic scenario that includes environmental uncertainties. For these experiments, ultrasound images were collected using SonoSite portable ultrasound system (SonoSite Inc., Bothell, WA) with a high frequency transducer. Passive markers were attached to the transducer and were tracked with a "Polaris" optical tracking system (Northern Digital Inc., Waterloo, Canada). For calibration, we used the standard crosswire technique [15]. About 500 ultrasound images were rapidly acquired in each experiment. The whole image acquisition took less than two minutes as the images were collected with a rate of about 10 frames per second. The dry pelvis bone was sank in water for collecting ultrasound images. The pixel size of the ultrasound images was $0.1 \mathrm{~mm}$.

For evaluation purposes, a CT scan of the whole pelvis was also acquired for each experiment with the slice thickness of $1 \mathrm{~mm}$. The CT scan was composed of about 260 slices of each 512 by 512 pixel (with the pixel size of $0.7 \mathrm{~mm}$ ). The pelvis was segmented in CT images using ANALYZE (Mayo Clinic, Rochester, Minn.). The marching cubes implementation in VTK was employed to create the mesh model of the pelvis. A ground truth can then be constructed by finding the CT-based pelvic coordinate system. Manual selection of landmarks in CT is pron to user error and subjective. In order to compute the CT-based coordinate system accurately and consistent with its definition in the atlas, the atlas was registered to the $\mathrm{CT}$ scan using a similar registration technique described in Section 2 (The average errors of this registration for dry bone, first cadaver, and second cadaver experiments were 1.4, 1.5, and $1.6 \mathrm{~mm}$ respectively). The anatomical coordinate system in the CT volume was transfered to the ultrasound coordinate system by registering the ultrasound points to the CT model (with average errors of 1.1, 1.6 and $1.4 \mathrm{~mm}$ for dry bone, first and second cadaver experiments, respectively). Despite the presence of registration errors, the CT-based coordinate is relatively accurate as long as there is no biased error introduced in the registration and the errors are distributed over a zero mean.

Assuming that the CT model is not available, the proposed method was used to recover the anatomical coordinate system using only the extracted ultrasound points and the Atlas. This coordinate system was compared to the CT-based coordinate system. Table 1 presents the results of comparison of the two coordinate systems for the cadaver experiments. In this table, $\alpha, \beta$, and $\gamma$ represent the angle between the axis of two coordinate system, and $x, y$, and $z$ show the error in locating the origin. Figure 3(a) demonstrates the result of localization of anatomical coordinate system in first cadaver experiment. The "global" reconstruction errors is highlighted in Figure 3(b) As expected, the error is lower for the regions from which data is collected. The atlas cannot accurately reconstruct sharp edges resulting at a higher error at the sharp edges. The average global reconstruction error is $3.3 \mathrm{~mm}$. The back part of pelvis is not compared since in the CT scan it was not segmented the same fashion as in the atlas. The results in Table 1 shows that this approach can accurately recover the orientation of the pelvis. This is because the registration accuracy in the regions of interest is 


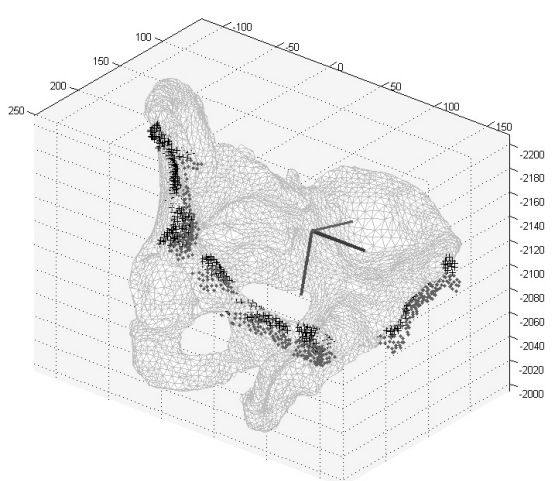

(a)

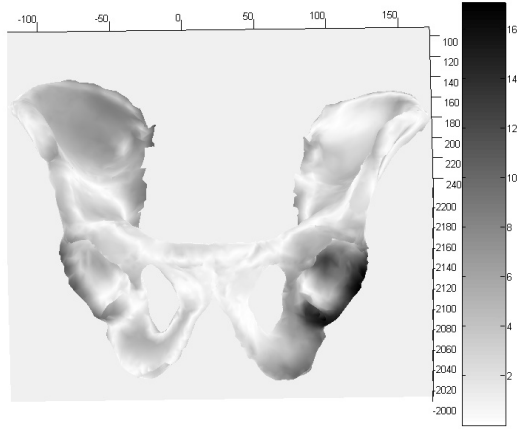

(b)

Fig. 3. Localization of the anatomical coordinate system in the first cadaver experiment. a) shows the reconstructed pelvis, the initial position of the extracted ultrasound points (denoted with dots), the registered position of them (denoted with crosses), and the calculated coordinate system. b) maps the combines error of reconstruction of pelvis to gray scale with dark representing higher errors.

high since the sample points are collected from these regions. The main sources of error are tracking/calibration accuracy and registration errors.

A 3.2 GHz P4 computer with 1 GB of RAM was used for all of the experiments. The method was implemented in MATLAB with some of the time-consuming functions written in $\mathrm{C}$ as a "mex function". With this setup, time for localization of coordinate system from the sample points was about two minutes.

\section{Conclusion and Future Work}

Our preliminary results show that our ultrasound based approach can localize the anatomical coordinate system of pelvis with an acceptable accuracy of about 1 degree. This is an inexpensive alternative which eliminates pre-operative CT and can be used in remote areas where other imaging modalities are not available. The atlas is shown to be an effective tool that can serve as a source of prior knowledge about the anatomical structure of the bone. It helps compensate for the missing data from the regions where ultrasound was not able to image. Finally, defining the coordinate system on the atlas allows for direct derivation of patient specific frame of reference from the results of registration.

As a future goal, the proposed method needs to be evaluated in a surgical scenario with real patients. A clinical trial can further approve reliability and effectiveness of this approach. For this purpose, the implementation should be optimized for speed and adjusted to the flow of surgery. It might also be possible to adapt a similar approach for other orthopedic applications such as knee replacement for navigation. 


\section{Acknowledgments}

The authors would like to thank Dr. Emad Boctor, and Hassan Rivaz for their help with cadaver experiments, as well as William R. Kenan, JR Fund Award for providing the cadavers. The authors would also like to thank SonoSite and Medtronics for the ultrasound imaging equipment. This work is supported in part by the National Science Foundation under grant EEC-9731478.

\section{References}

1. Honl, M., et al.: Orientation of the acetabular component: A comparison of five navigation systems with conventional surgical technique. Journal of Bone and Joint Surgery 88(B(10)), 1401-1405 (2006)

2. Mccollum, D.E., Gray, W.J.: Dislocation after total hip arthroplasty causes and prevention. Clinical Orthopaedics \& Related Research 261, 159-170 (1990)

3. Keppler, P., et al.: A new experimental measurement and planning tool for sonographic-assisted navigation. Orthopedics 30(10), S144-147 (2007)

4. Kiefer, H., Othman, A.: Ultrasound vs pointer palpation based method in THA navigation: a comparative study. Orthopedics 30(10), S153-156 (2007)

5. Cootes, T.F., Taylor, C.J.: Active appearance models. World Wide Web Publication - Technical report (March 2004)

6. Talib, H., Rajamani, K., Kowal, J., Styner, M., Ballester, M.A.G.: Assessing the feasibility of ultrasound-initialized deformable bone models. In: Proceedings of SPIE Medical Imaging (2006)

7. Zheng, G., et al.: Accurate and robust reconstruction of a surface model of the proximal femur from sparse-point data and a dense-point distribution model for surgical navigation. IEEE Tran. Biomed. Eng. 54(12), 2109-2122 (2007)

8. Chan, C.S.K., Edwards, P.J., Hawkes, D.J.: Integration of ultrasound-based registration with statistical shape models for computer-assisted orthopaedic surgery. In: Proceedings of SPIE, pp. 1605-7422 (2003)

9. Chan, C.S.K., et al.: Cadaver validation of the use of ultrasound for 3D model instantiation of bony anatomy in image guided orthopaedic surgery. In: Barillot, C., Haynor, D.R., Hellier, P. (eds.) MICCAI 2004. LNCS, vol. 3217, pp. 397-404. Springer, Heidelberg (2004)

10. Nikou, C., Jaramaz, B., DiGioia, A.M., Levison, T.J.: Description of anatomic coordinate systems and rationale for use in an image-guided total hip replacement system. In: Delp, S.L., DiGoia, A.M., Jaramaz, B. (eds.) MICCAI 2000. LNCS, vol. 1935, pp. 1188-1194. Springer, Heidelberg (2000)

11. Foroughi, P., Boctor, E., Schwartz, M.J., Taylor, R.H., Fichtinger, G.: Ultrasound bone segmentation using dynamic programming. In: IEEE Ultrasonics Symposium, pp. 2523-2526 (2007)

12. Chintalapani, G., Ellingsen, L.M., Sadowsky, O., Prince, J.L., Taylor, R.H.: Statistical atlases of bone anatomy: Construction, iterative improvement and validation. In: Ayache, N., Ourselin, S., Maeder, A. (eds.) MICCAI 2007, Part I. LNCS, vol. 4791, pp. 499-506. Springer, Heidelberg (2007)

13. Foroughi, P., Taylor, R.H., Fichtinger, G.: Automatic initialization for 3d bone registration. In: Proceedings of SPIE Medical Imaging, 69182P (2007) 
14. Yao, J., Taylor, R.H.: Assessing accuracy factors in deformable 2D/3D medical image registration using a statistical pelvis model. In: Proceedings of IEEE International Conference on Computer Vision, pp. 1329-1334 (2003)

15. Prager, R.W., Rohling, R.N., Gee, A.H., Berman, L.: Rapid calibration for 3-D freehand ultrasound. Ultrasound in Medicine and Biology 24(6), 855-869 (1998) 